\title{
A DETERMINISTIC INVENTORY/PRODUCTION MODEL WITH GENERAL InVENTORY Cost RATE FunCtion and Concave PRoduction Costs
}

\author{
Z.P. Bayındır, S.I. Birbil and J.B.G. Frenk
}

\begin{tabular}{|l|l|}
\hline \multicolumn{2}{|l|}{ ERIM REPORT SERIES RESEARCH IN MANAGEMENT } \\
\hline ERIM Report Series reference number & ERS-2004-064-LIS \\
\hline Publication & July 2004 \\
\hline Number of pages & 13 \\
\hline Email address corresponding author & sibirbil@few.eur.nl \\
\hline Address & Erasmus Research Institute of Management (ERIM) \\
& Rotterdam School of Management / Rotterdam School of \\
& Economics \\
& Erasmus Universiteit Rotterdam \\
& P.O. Box 1738 \\
& 3000 DR Rotterdam, The Netherlands \\
& Phone: +31 10 408 1182 \\
& Fax: +31 10 408 9640 \\
& Email: info@erim.eur.nl \\
& Internet: www.erim.eur.nl \\
\hline
\end{tabular}

Bibliographic data and classifications of all the ERIM reports are also available on the ERIM website: www.erim.eur.nl 


\title{
ERASMUS RESEARCH INSTITUTE OF MANAGEMENT
}

\author{
REPORT SERIES \\ RESEARCH IN MANAGEMENT
}

\begin{tabular}{|c|c|c|}
\hline \multicolumn{3}{|c|}{ BIBLIOGRAPHIC DATA AND CLASSIFICATIONS } \\
\hline Abstract & \multicolumn{2}{|c|}{$\begin{array}{l}\text { We present a thorough analysis of the economic order quantity model with shortages under a } \\
\text { general inventory cost rate function and concave production costs. By using some standard } \\
\text { results from convex analysis, we show that the model exhibits a composite concave-convex } \\
\text { structure. Consequently, an effective solution procedure, particularly useful for an approximation } \\
\text { scheme, is proposed. A computational study is appended to illustrate the performance of the } \\
\text { proposed solution procedure. }\end{array}$} \\
\hline \multirow{3}{*}{$\begin{array}{l}\text { Library of Congress } \\
\text { Classification } \\
\text { (LCC) }\end{array}$} & $5001-6182$ & Business \\
\hline & $5201-5982$ & Business Science \\
\hline & HB 143 & Mathematical Programming \\
\hline \multirow{4}{*}{$\begin{array}{l}\text { Journal of Economic } \\
\text { Literature } \\
\text { (JEL) }\end{array}$} & M & Business Administration and Business Economics \\
\hline & M 11 & Production Management \\
\hline & R 4 & Transportation Systems \\
\hline & C 6 & Mathematical Methods and Programming \\
\hline \multirow{4}{*}{$\begin{array}{l}\text { European Business Schools } \\
\text { Library Group } \\
\text { (EBSLG) }\end{array}$} & $85 \mathrm{~A}$ & Business General \\
\hline & $260 \mathrm{~K}$ & Logistics \\
\hline & $240 \mathrm{~B}$ & Information Systems Management \\
\hline & $255 B$ & Decisions under constraints, Programming \\
\hline \multicolumn{3}{|c|}{ Gemeenschappelijke Onderwerpsontsluiting (GOO) } \\
\hline \multirow[t]{4}{*}{ Classification GOO } & 85.00 & Bedrijfskunde, Organisatiekunde: algemeen \\
\hline & 85.34 & Logistiek management \\
\hline & 85.20 & Bestuurlijke informatie, informatieverzorging \\
\hline & 85.03 & Methoden en technieken, operations research \\
\hline \multirow[t]{3}{*}{ Keywords GOO } & \multicolumn{2}{|c|}{ Bedrijfskunde / Bedrijfseconomie } \\
\hline & \multicolumn{2}{|c|}{ Bedrijfsprocessen, logistiek, management informatiesystemen } \\
\hline & \multicolumn{2}{|c|}{ Voorraadbeheer, kosten, wiskundige methoden } \\
\hline Free keywords & \multicolumn{2}{|c|}{$\begin{array}{l}\text { Inventory, general inventory cost function, concave production cost, concave-convex } \\
\text { programming }\end{array}$} \\
\hline
\end{tabular}




\title{
A Deterministic Inventory/Production Model with General Inventory Cost Rate Function and Concave Production Costs
}

\author{
Z. P. Bayındır ${ }^{\dagger}$, Ş. İ. Birbil ${ }^{\ddagger}$ and J. B. G. Frenk $k^{\dagger *}$ \\ ${ }^{\dagger}$ Econometric Institute, Erasmus University Rotterdam \\ Postbus 1738, 3000 DR Rotterdam, The Netherlands. \\ \{bayindir, frenk\}@few.eur.nl \\ ${ }^{\ddagger}$ Erasmus Research Institute of Management, Erasmus University Rotterdam \\ Postbus 1738, 3000 DR Rotterdam, The Netherlands. \\ sibirbilefew.eur.nl
}

\begin{abstract}
We present a thorough analysis of the economic order quantity model with shortages under a general inventory cost rate function and concave production costs. By using some standard results from convex analysis, we show that the model exhibits a composite concave-convex structure. Consequently, an effective solution procedure, particularly useful for an approximation scheme, is proposed. A computational study is appended to illustrate the performance of the proposed solution procedure.
\end{abstract}

Keywords. inventory, general inventory cost function, concave production cost, concave-convex programming

\section{Introduction}

In inventory control theory the economic order quantity (EOQ) model is the most fundamental model, which dates back to the pioneering work of Harris [1]. The environment of the model is somehow restricted. The demand rate is known and constant, shortages are not permitted, there is a fixed setup cost, and the unit purchasing and holding costs are independent of the size of the replenishment order. In this simplest form, the model describes the tradeoff between the fixed setup cost and the holding cost. A natural extension of this model to a production environment deals with the case of a finite production rate and associated fixed unit production cost. This model is known as the economic production quantity (EPQ) model [2, 3].

The present article discusses a deterministic production model. Certain assumptions of the EPQ model are preserved; such as, a fixed setup cost of $a>0$, stationary and deterministic demand with rate $\lambda>0$, finite and deterministic production with rate $\mu>\lambda$. However, the following extensions on the costing scheme are considered:

$\diamond$ The variable production cost of the item is not necessarily linear but given by a concave production

\footnotetext{
${ }^{*}$ Corresponding author.
} 
cost function. This reflects the economies of scale situation; that is, the marginal cost of each unit produced decreases as the lot size increases. Cost of producing a lot of size $Q$ is $c(Q)$ with $c(\cdot)$ being a continuous, strictly increasing concave function satisfying $c(0)=0$ and $c(\infty)=\infty$.

$\diamond$ The inventory holding cost consists of two components:

- Unit out-of-pocket holding cost includes, for example, the real costs like the insurance cost and the warehouse rent. This holding cost component is given by a strictly increasing function, which is not necessarily linear in the amount of inventory.

- Unit opportunity cost of holding reflects the opportunity cost of investing into inventories. We consider unit production cost as the cost added to each item. Since unit production cost depends on the lot size, the inventory value of each item in a certain lot is not identical. We utilize the average costing principle. Therefore, under the traditional way of setting holding cost rates when the inventory carrying charge is $r$, an opportunity cost of $\frac{c(Q)}{Q} r$ is incurred per unit time for an item produced in a lot of size $Q$.

$\diamond$ Unfilled demand is assumed to be completely backordered. The penalty cost of backordering is not necessarily linear but strictly increasing in the backordered amount.

We consider an $(S, T)$ type of inventory control rule. According to this rule, the net inventory level is raised up-to level $S$ at every $T$ time units. Since we adopt complete backordering, a production order of $\lambda T$ units is given at every $T$ time periods. The net inventory level under this policy is illustrated in Figure 1.

Under the considered costing scheme, the inventory (backorder) cost rate function in a cycle length of $T$ is given by

$$
f(z, T):= \begin{cases}f_{1}(z)+\frac{c(\lambda T) r}{\lambda T} z & \text { if } z \geq 0 \\ f_{1}(z) & \text { if } z<0,\end{cases}
$$

with the function $f_{1}(\cdot)$ decreasing on $(-\infty, 0)$ (backorder penalty) and strictly increasing on $(0, \infty)$ (outof-pocket holding cost). We further assume that the function $f_{1}(\cdot)$ is continuous and satisfies $f_{1}(0)=0$. We acknowledge that a similar costing structure is considered by Porteus within stochastic inventory models [4].

Using Figure 1, the total inventory and penalty cost in one cycle of length $T>0$ is given by

$$
\int_{0}^{\bar{T}} f(S-\lambda t, T) d t+\int_{0}^{T-\bar{T}} f(S-(\mu-\lambda) t, T) d t
$$

where $\bar{T}:=\left(1-\frac{\lambda}{\mu}\right) T$. After some simple calculations and defining the auxiliary parameter $\sigma:=\lambda\left(1-\frac{\lambda}{\mu}\right)$, one can show that the total inventory and penalty cost for $T>0$ and $0 \leq S \leq \sigma T$ is equal to

$$
\int_{0}^{T} f(S-\sigma t, T) d t=\int_{0}^{T} f_{1}(S-\sigma t) d t+\frac{c(\lambda T) r S^{2}}{2 \lambda T \sigma}
$$

Consequently, if we take into account the setup and production costs, then the average cost $A(S, T)$ under the $(S, T)$ inventory control rule is given by

$$
A(S, T):=\frac{a+c(\lambda T)+\frac{c(\lambda T) r S^{2}}{2 \lambda T \sigma}+\int_{0}^{T} f_{1}(S-\sigma t) d t}{T} .
$$

The main objective is now to determine the optimal policy parameters $(S, T)$ by solving the optimization problem

$$
v_{P}:=\min _{T>0,0 \leq S \leq \sigma T} A(S, T) .
$$




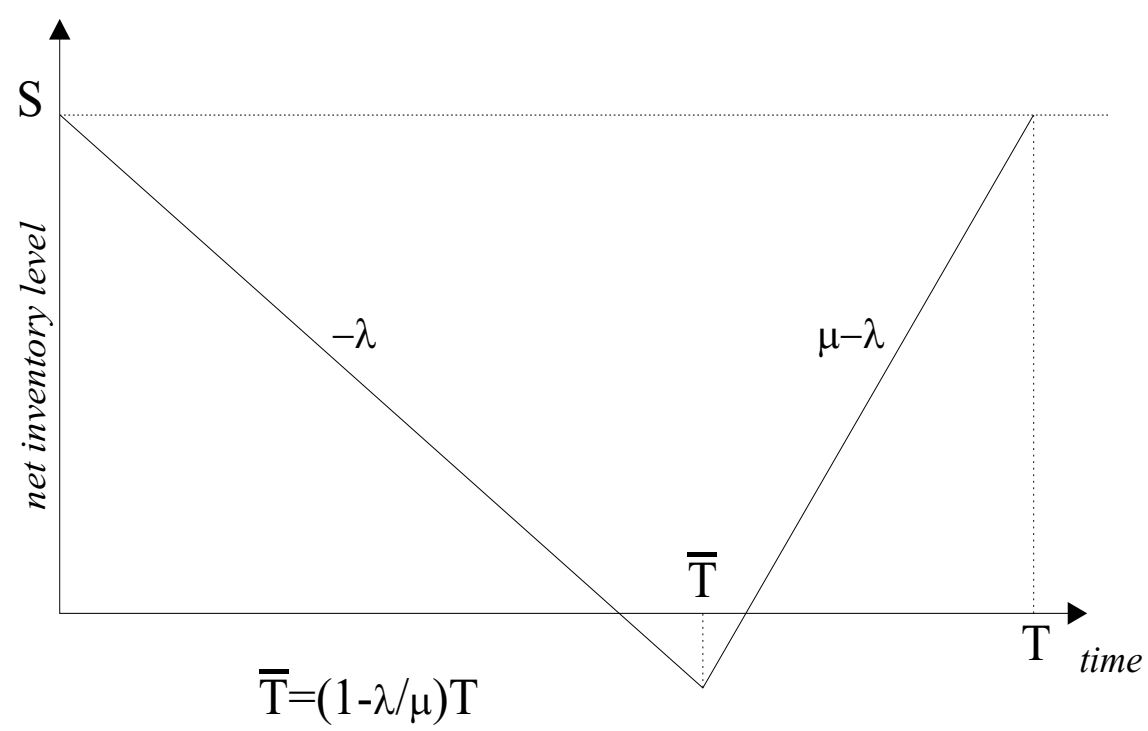

Figure 1: Inventory level for the item over time.

In this paper, we aim at analyzing the structure of optimization problem $(\mathrm{P})$ and identifying a class of cost rate functions $f_{1}(\cdot)$ and $c(\cdot)$, under which it is relatively easy to find the optimal global solution. Our main contributions are the following:

$\diamond$ Investigation of an extended production/inventory model under concave production cost and general inventory cost rate function.

$\diamond$ Reformulation of the extended model by using convex analysis.

$\diamond$ Developing tailor-made algorithms for the solution of the model, when the out-of-pocket holding cost function is piecewise linear and/or the production cost function is polyhedral concave.

$\diamond$ Discussing the implementation details as well as providing some computational results.

The outline of the paper is as follows. In Section 2 the structure of the considered optimization problem is analyzed. An important special case of the model with piecewise linear and/or polyhedral concave functions is discussed in Section 3. Finally we explain, in Section 4, our computational experience with the proposed algorithm.

\section{The Structure of Optimization Problem (P)}

This section includes our analysis of optimization problem $(\mathrm{P})$. It is important to notice that optimization problem (P) is separable with respect to the decision variables $S$ and $T$. Therefore, if we denote the optimal inventory holding and penalty cost in a cycle length of $T$ with the function $\phi:(0, \infty) \rightarrow \mathbb{R}$ given by

$$
\phi(T):=\min _{0 \leq S \leq \sigma T}\left\{\frac{c(\lambda T) r S^{2}}{2 \lambda T \sigma}+\int_{0}^{T} f_{1}(S-\sigma t) d t\right\},
$$

then problem $(\mathrm{P})$ can be rewritten as

$$
v_{P}=\min _{T>0} \Phi(T),
$$


where the function $\Phi:(0, \infty) \rightarrow \mathbb{R}$ corresponds to

$$
\Phi(T):=\frac{a+c(\lambda T)+\phi(T)}{T} .
$$

To investigate the structure of the objective function $\Phi(\cdot)$, we introduce the function $F:[0, \infty) \times$ $(0, \infty) \rightarrow \mathbb{R}$ given by

$$
F(x, T):=\frac{a+x+\min _{0 \leq S \leq \sigma T}\left\{\frac{x r S^{2}}{2 \lambda T \sigma}+\int_{0}^{T} f_{1}(S-\sigma t) d t\right\}}{T} .
$$

It is easy to check that

$$
F(x, T)=\min _{0 \leq S \leq \sigma T}\left\{\frac{a+x+\frac{x r S^{2}}{2 \lambda T \sigma}+\int_{0}^{T} f_{1}(S-\sigma t) d t}{T}\right\} .
$$

Since for every $T>0$ the function $x \rightarrow F(x, T)$ is the minimum of a sequence of increasing affine functions, $F(\cdot, T)$ is increasing and concave. We obtain by the definition of $\Phi(\cdot)$ that

$$
\Phi(T)=F(c(\lambda T), T)
$$

and hence,

$$
v_{P}=\min _{T>0} F(c(\lambda T), T) .
$$

Using now (2.3), one can show the following result, where $c_{-}^{\prime}(\cdot)$ and $c_{+}^{\prime}(\cdot)$ denote the left and right derivatives of the function $c(\cdot)$, respectively.

Lemma 2.1 If $c^{*}(\cdot)$ is given by

$$
c^{*}(\omega):=\min _{T>0}\{\omega T-c(T)\}
$$

for $\omega \in \Omega:=\left[c_{-}^{\prime}(\infty), c_{+}^{\prime}(0)\right]$, then it follows that

$$
v_{P}=\min _{\omega \in \Omega} \min _{T>0} F\left(\lambda T \omega-c^{*}(\omega), T\right)
$$

Proof. Since the production cost function $c(\cdot)$ is an increasing continuous concave function satisfying $c(0)=0$ and $c(\infty)=\infty$, it follows for every $T>0$ that (see Appendix A in [5])

$$
c(\lambda T)=\min _{\omega \in \Omega}\left\{\lambda T \omega-c^{*}(\omega)\right\} .
$$

Note that for every $T>0$, the function $x \rightarrow F(x, T)$ is increasing on $(0, \infty)$. Therefore, by relation (2.2) we can write

$$
\Phi(T)=F\left(\min _{\omega \in \Omega}\left\{\lambda T \omega-c^{*}(\omega)\right\}, T\right)=\min _{\omega \in \Omega} F\left(\lambda T \omega-c^{*}(\omega), T\right) .
$$

Using now relation (2.3) leads to

$$
\begin{aligned}
v_{P} & =\min _{T>0} \min _{\omega \in \Omega} F\left(\lambda T \omega-c^{*}(\omega), T\right) \\
& =\min _{\omega \in \Omega} \min _{T>0} F\left(\lambda T \omega-c^{*}(\omega), T\right) .
\end{aligned}
$$

This shows the desired result.

We next show that the objective function of the above inventory problem is a composition of concave and convex functions. Therefore the problem is actually a C-programming problem [6]. Sniedovich introduces this subclass of global optimization problems and proposes a parametric approach to solve 
these problems. Since a stationary point is not necessarily a global optimal solution for these problems, the parametric approach or some other classical nonlinear programming techniques might have difficulty in identifying the exact global optimal solution. To verify that problem $(\mathrm{P})$ is a C-programming problem, we introduce the function $\varphi: \mathbb{R}_{+}^{3} \rightarrow \mathbb{R}$ given by

$$
\varphi(x, y, T):=\min _{0 \leq S \leq \sigma T}\left\{\left(\frac{x}{2 \sigma}+\frac{y}{2 \lambda T \sigma}\right) r S^{2}+\int_{0}^{T} f_{1}(S-\sigma t) d t\right\},
$$

and the function $v: \mathbb{R}_{+}^{2} \rightarrow \mathbb{R}$ is given by

$$
v(x, y):=\min _{T>0} \frac{a+y+\varphi(x, y, T)}{T} .
$$

After some calculations, it follows that

$$
\min _{T>0} F\left(\lambda \omega T-c^{*}(\omega), T\right)=v\left(\omega,-c^{*}(\omega)\right)+\lambda \omega,
$$

and this implies by Lemma 2.1 that

$$
v_{P}=\min _{\omega \in \Omega}\left\{v\left(\omega,-c^{*}(\omega)\right)+\lambda \omega\right\} .
$$

Since for every $T>0$, the function $(x, y) \rightarrow \varphi(x, y, T)$ is concave on $\mathbb{R}_{+}^{2}$, we obtain by relation (2.6) that the function $v(\cdot, \cdot)$ is also concave on $\mathbb{R}_{+}^{2}$. Using now the representation listed in relation (2.8), it is easy to verify our claim that the objective function of problem $(\mathrm{P})$ is a composition of concave and convex functions (note that $c^{*}(\cdot)$ is concave).

Due to the complexity of finding a global optimal solution to problem (P), we consider in the next example an important class of concave production cost functions for which the structure of the problem given in Lemma 2.1 can be simplified.

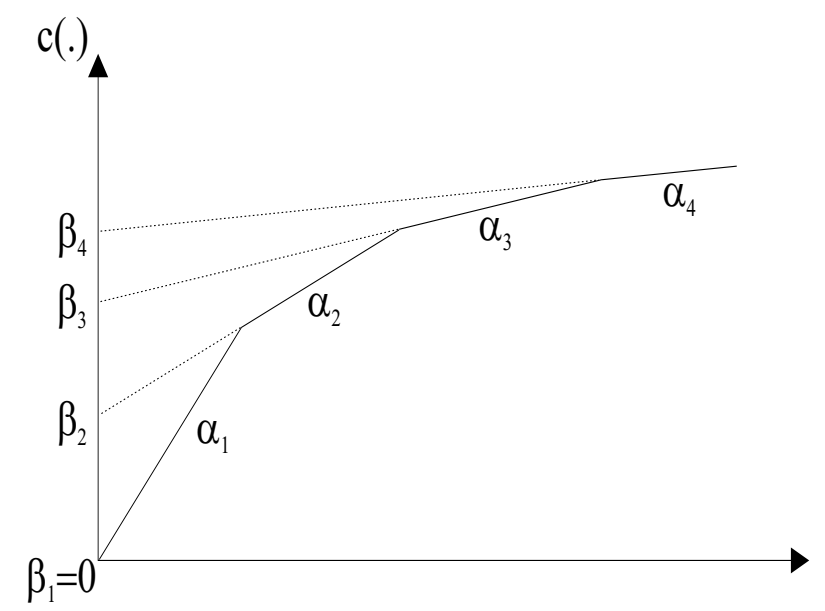

Figure 2: An example polyhedral concave function $c(\cdot)$ where $m=4$.

Example 2.1 Let the production cost function $c(\cdot)$ be a polyhedral concave function given by

$$
c(Q)=\min _{1 \leq j \leq m}\left\{\alpha_{j} Q+\beta_{j}\right\},
$$

where $\alpha_{m}<\alpha_{m-1}<\cdots<\alpha_{1}$ and $0=\beta_{1}<\beta_{2}<\cdots<\beta_{m}$ (see Figure 2). For the function listed in (2.9), it is easy to check that $\Omega=\left[\alpha_{m}, \alpha_{1}\right]$, and since $c^{*}(\cdot)$ is a polyhedral concave function with 
breaking points $\alpha_{j}, 1 \leq j \leq m$, we obtain that the function $\omega \rightarrow \lambda \omega T-c^{*}(\omega)$ is a polyhedral convex function with breaking points $\alpha_{j}, 1 \leq j \leq m$. This means that the function $\omega \rightarrow \lambda \omega T-c^{*}(\omega)$ is linear on $\left[\alpha_{j}, \alpha_{j-1}\right], 2 \leq j \leq m$. Thus, by the concavity of the function $x \rightarrow F(x, T)$ this yields for every $T>0$ that

$$
\min _{\omega \in \Omega} F\left(\lambda \omega T-c^{*}(\omega), T\right)=\min _{1 \leq j \leq m} F\left(\lambda \alpha_{j} T-c^{*}\left(\alpha_{j}\right), T\right) .
$$

Using now $c^{*}\left(\alpha_{j}\right)=-\beta_{j}$, we obtain

$$
\min _{\omega \in \Omega} F\left(\lambda \omega T-c^{*}(\omega), T\right)=\min _{1 \leq j \leq m} F\left(\lambda \alpha_{j} T+\beta_{j}, T\right) .
$$

This shows by relation (2.4) that

$$
v_{P}=\min _{1 \leq j \leq m} \min _{T>0} F\left(\lambda \alpha_{j} T+\beta_{j}, T\right) .
$$

Equivalently, by using relation (2.8) we can write

$$
v_{P}=\min _{1 \leq j \leq m}\left\{v\left(\alpha_{j}, \beta_{j}\right)+\lambda \alpha_{j}\right\}
$$

In the above example, we only need to solve $m$ optimization problems. In the more general setting, as considered in Lemma 2.1, we need to solve for every $\omega \in \Omega$, the inner optimization problem

$$
\min _{T>0} F\left(\lambda \omega T-c^{*}(\omega), T\right)
$$

It is obvious that the decision variable $T$ in this optimization problem can be replaced by $T^{-1}$ without changing the optimal objective function value. This is an important observation, since after this simple transformation, as shown in the next lemma, the inner optimization problem

$$
\min _{T>0} F\left(\lambda \omega T^{-1}-c^{*}(\omega), T^{-1}\right)
$$

becomes a convex optimization problem.

Lemma 2.2 For each $\omega \in \Omega$, the optimization problem $(P(\omega))$ is a convex optimization problem.

Proof. It is shown in Lemma 3.2 of [7] that the function

$$
(T, S) \rightarrow \int_{0}^{T} f_{1}(S-\sigma t) d t
$$

is convex on the convex cone $K:=\{(T, S): T>0,0 \leq S \leq \sigma T\}$. Moreover, applying Theorem 5.16 of [8], it follows for every $\omega \in \Omega$ and using $c^{*}(\omega) \leq 0$ that the function

$$
(T, S) \rightarrow r\left(\frac{\omega}{2 \sigma}-\frac{c^{*}(\omega)}{2 \lambda \sigma T}\right) S^{2}
$$

is convex on $\mathbb{R}_{+}^{2}$. These observations imply that the function

$$
(T, S) \rightarrow r\left(\frac{\omega}{2 \sigma}-\frac{c^{*}(\omega)}{2 \lambda \sigma T}\right) S^{2}+\int_{0}^{T} f_{1}(S-\sigma t) d t
$$

is convex on $K$. By a similar proof as done in Theorem 3.2 of [7], we obtain that the function

$$
T \rightarrow \min _{0 \leq S \leq \sigma T}\left\{r\left(\frac{\omega}{2 \sigma}-\frac{c^{*}(\omega)}{2 \lambda \sigma T}\right) S^{2}+\int_{0}^{T} f_{1}(S-\sigma t) d t\right\}
$$


is convex on $(0, \infty)$. This shows that the function

$$
T \rightarrow a-c^{*}(\omega)+\min _{0 \leq S \leq \sigma T}\left\{r\left(\frac{\omega}{2 \sigma}-\frac{c^{*}(\omega)}{2 \lambda \sigma T}\right) S^{2}+\int_{0}^{T} f_{1}(S-\sigma t) d t\right\}
$$

is also convex on $(0, \infty)$. It can now be shown for any function $g:(0, \infty) \rightarrow \mathbb{R}$ that $T \rightarrow g(T)$ is convex on $(0, \infty)$ if and only if $T \rightarrow T g\left(T^{-1}\right)$ is convex on $(0, \infty)$ [9]. Applying this result to the function

$$
g(T):=a-c^{*}(\omega)+\min _{0 \leq S \leq \sigma T}\left\{r\left(\frac{\omega}{2 \sigma}-\frac{c^{*}(\omega)}{2 \lambda \sigma T}\right) S^{2}+\int_{0}^{T} f_{1}(S-\sigma t) d t\right\}
$$

and using

$$
F\left(\lambda \omega T^{-1}-c^{*}(\omega), T^{-1}\right)-\lambda \omega=T g\left(T^{-1}\right)
$$

the desired result follows.

\section{Optimization Problem (P) with Piecewise Linear Functions}

As we have already discussed, finding a global optimal solution of the general problem is quite difficult due to the composite concave-convex structure. Therefore, it seems reasonable to consider special cases of this problem to which one can apply standard optimization packages that return an approximation of a global optimal solution.

We have already discussed in Example 2.1 the structure of the optimization problem (see relation (2.10)) when $c(\cdot)$ is a polyhedral concave function. In practice the production cost functions with a number of breaking points frequently occur. For instance, when incremental discounting is applied, the cost function is represented exactly by the polyhedral concave function [3]. In other cases, we can always approximate any continuous concave production cost function by a polyhedral concave cost function. This means by Lemma 2.2 that we only need to solve a finite number of convex programming problems. Therefore, it seems that we can use a standard convex programming solver. However, the objective function of this convex programming problem involves the evaluation of an integral, which could be approximated by a numerical procedure. If we use the simple trapezoidal algorithm [10], this evaluation boils down to replacing a cost rate function $f_{1}(\cdot)$ (with linear backorder cost) by a piecewise linear function given by

$$
f_{1}(z):= \begin{cases}-p z & \text { for } z<0 \\ h_{1} z & \text { for } 0 \leq z<u_{1} \\ \vdots & \\ b_{i-1}+h_{i}\left(z-u_{i-1}\right) & \text { for } u_{i-1} \leq z<u_{i} \\ \vdots & \\ b_{n-1}+h_{n}\left(z-u_{n-1}\right) & \text { for } u_{n-1} \leq z<\infty\end{cases}
$$

where $b_{i}=f_{1}\left(u_{i}\right)$ for $1 \leq i \leq n-1$ (see Figure 3 ).

At this point we assume that the cost rate function is represented by a piecewise linear function, and in the subsequent discussion we study the details of a tailor made algorithm for this class of functions. 


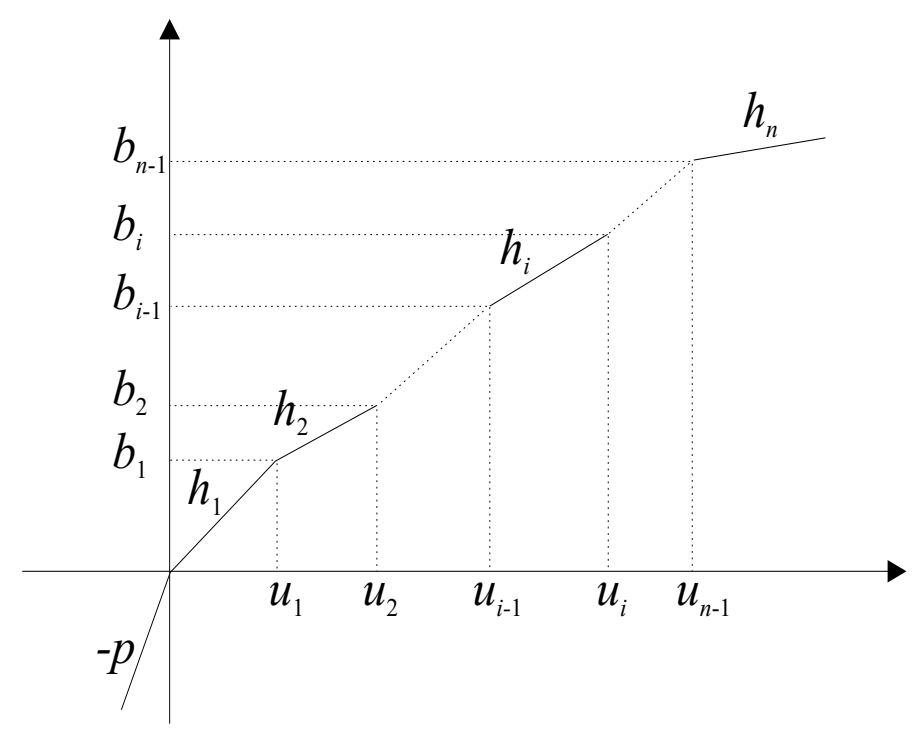

Figure 3: Piecewise linear cost rate function.

To construct such an algorithm, we first need to analyze for every $(x, y) \in \mathbb{R}_{+}^{2}$ the optimization problem listed in (2.5). It easy to verify that the associated objective function

$$
\psi(x, y, T, S):=\left(\frac{x}{2 \sigma}+\frac{y}{2 \lambda T \sigma}\right) r S^{2}+\int_{0}^{T} f_{1}(S-\sigma t) d t
$$

satisfies

$$
\begin{aligned}
\frac{\partial \psi}{\partial S}(x, y, T, S) & =\left(x+\frac{y}{\lambda T}\right) \frac{r S}{\sigma}+\frac{1}{\sigma}\left(f_{1}(S)-f_{1}(S-\sigma T)\right) \\
& =\left(x+\frac{y}{\lambda T}\right) \frac{r S}{\sigma}+\frac{1}{\sigma}\left(f_{1}(S)+p(S-\sigma T)\right),
\end{aligned}
$$

for every $0<S<\sigma T$ and $T>0$. This shows for any positive $x, y, T$ that the function $S \rightarrow$ $\frac{\partial \psi}{\partial S}(x, y, T, S)$ is strictly increasing and continuous on $(0, \sigma T)$. Moreover, since

$$
\frac{\partial \psi}{\partial S}(x, y, T, 0)=\lim _{S \downarrow 0} \frac{\partial \psi}{\partial S}(x, y, T, S)=-p T<0
$$

and

$$
\begin{aligned}
\frac{\partial \psi}{\partial S}(x, y, T, \sigma T) & =\lim _{S \uparrow \sigma T} \frac{\partial \psi}{\partial S}(x, y, T, S) \\
& =\left(x+\frac{y}{\lambda T} r T\right)+\frac{1}{\sigma} f_{1}(\sigma T)>0,
\end{aligned}
$$

we obtain for every positive $x, y, T$ that the optimal solution of the optimization problem

$$
\min _{0 \leq S \leq \sigma T}\left\{\left(\frac{x}{2 \sigma}+\frac{y}{2 \lambda T \sigma}\right) r S^{2}+\int_{0}^{T} f_{1}(S-\sigma T) d t\right\}
$$

is unique and belongs to $(0, \sigma T)$. Denoting this optimal solution by $S(x, y, T)$, it follows by relation (3.3) and the previous observations that the value of $S(x, y, T)$ is the unique solution of the system

$$
\left(x+\frac{y}{\lambda T}\right) r S+f_{1}(S)+p(S-\sigma T)=0,0<S<\sigma T .
$$

This shows that the function $T \rightarrow S(x, y, T)$ is continuous, strictly increasing and satisfies $\lim _{T \uparrow \infty} S(x, y, T)=$ $\infty$. Hence for every $1 \leq i \leq n-1$, there exists a unique $T_{i}(x, y)>0$ such that

$$
S\left(x, y, T_{i}(x, y)\right)=u_{i}
$$


To write down an analytical expression for $T_{i}(x, y)$ we observe by relation (3.4) that

$$
\left(x+\frac{y}{\lambda T_{i}(x, y)}\right) r u_{i}+b_{i}+p\left(u_{i}-\sigma T_{i}(x, y)\right)=0 .
$$

This implies

$$
T_{i}^{2}(x, y)-d_{i} T_{i}(x, y)-e_{i}=0
$$

with

$$
d_{i}:=\frac{1}{\sigma p}\left(r u_{i} x+b_{i}+p u_{i}\right)>0 \text { and } e_{i}:=\frac{y r u_{i}}{\lambda \sigma p}>0,
$$

and due to $T_{i}(x, y)>0$, this shows that

$$
T_{i}(x, y)=\frac{d_{i}}{2}+\frac{1}{2} \sqrt{4 e_{i}+d_{i}^{2}}
$$

for every $1 \leq i \leq n-1$. By the monotonicity of the function $T \rightarrow S(x, y, T)$, we obtain with $u_{0}:=0$, $b_{0}:=0$, and hence $T_{0}(x, y):=0$, that

$$
u_{i-1} \leq S(x, y, T)<u_{i}
$$

for every $T_{i-1}(x, y) \leq T<T_{i}(x, y)$ and $1 \leq i \leq n-1$ (see Figure 4 ). In particular one can show the following result.

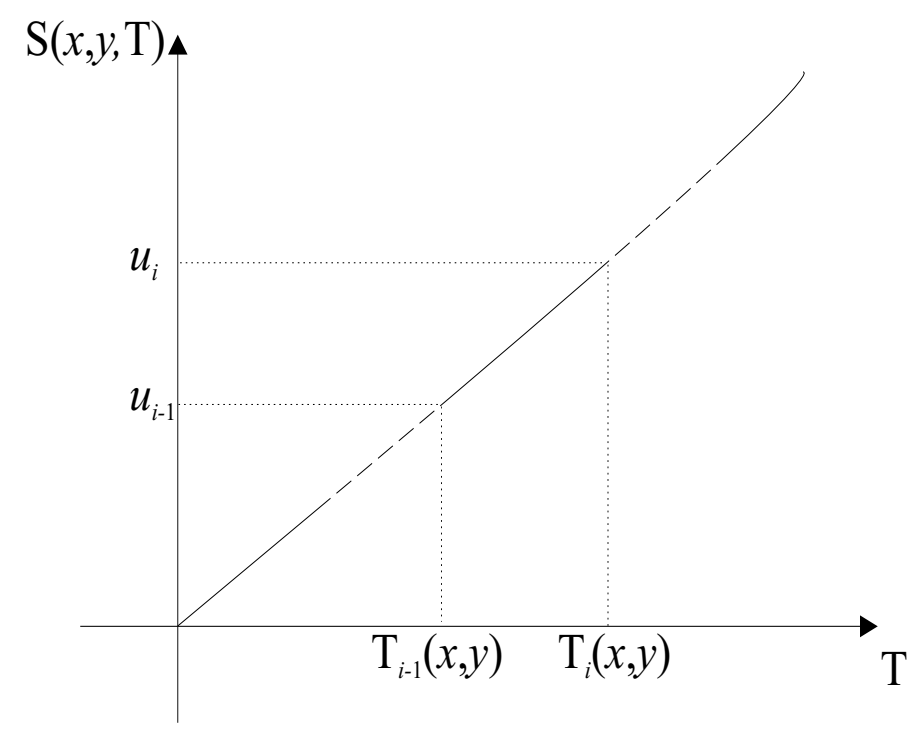

Figure 4: Calculation of $S(x, y, T)$.

Lemma 3.1 For every $T_{i-1}(x, y) \leq T<T_{i}(x, y), 1 \leq i \leq n-1$, the unique optimal solution $S(x, y, T)$ of the optimization problem (2.5) is given by

$$
S(x, y, T)=\frac{\left(h_{i} u_{i-1}-b_{i-1}\right) T+p \sigma T^{2}}{\left(r x+h_{i}+p\right) T+r y \lambda^{-1}} .
$$

Proof. If the value $T$ satisfies $T_{i-1}(x, y) \leq T<T_{i}(x, y)$, then we obtain by relation (3.4) that

$$
\left(x+\frac{y}{\lambda T}\right) r S(x, y, T)+f_{1}(S(x, y, T))+p(S(x, y, T)-\sigma T)=0 .
$$


Using now relations (3.1) and (3.6), we know that

$$
f_{1}(S(x, y, T))=b_{i-1}+h_{i}\left(S(x, y, T)-u_{i-1}\right) .
$$

Therefore, by combining both relations the desired result follows.

Since we know by the definition of $S(x, y, T)$ that

$$
\frac{\partial \psi}{\partial S}(x, y, T, S(x, y, T))=0
$$

we obtain by standard calculus arguments that

$$
\begin{aligned}
\frac{\partial \varphi}{\partial T}(x, y, T) & =\frac{\partial \psi}{\partial T}(x, y, T, S(x, y, T)) \\
& =\frac{-y r S^{2}(x, y, T)}{2 \lambda T^{2} \sigma}+f_{1}(S(x, y, T)-\sigma T)
\end{aligned}
$$

This implies by the structure of $f_{1}(\cdot)$ that

$$
\frac{\partial \varphi}{\partial T}(x, y, T)=\frac{-y r S^{2}(x, y, T)}{2 \lambda T^{2} \sigma}+p(\sigma T-S(x, y, T)),
$$

which is easy to calculate by Lemma 3.1. In a similar way as we have done in Lemma 2.2, it can be shown that $T \rightarrow \varphi(x, y, T)$ is convex in $(0, \infty)$. Consequently, if we replace the decision variable $T$ by $T^{-1}$, then the optimization problem

$$
v(x, y)=\min _{T>0}\left\{(a+y) T+T \varphi\left(x, y, T^{-1}\right)\right\}
$$

becomes a convex optimization problem. This implies for the optimal solution $T^{*}(x, y)$ of the optimization problem (2.6) that the associated objective function

$$
T \rightarrow \frac{a+y+\varphi(x, y, T)}{T}
$$

is increasing for $T \geq T^{*}(x, y)$ and decreasing for $T \leq T^{*}(x, y)$. To compute $T^{*}(x, y)$, we first take the derivative of the above function with respect to $T$ and then solve the system

$$
-T^{-2}(a+y+\varphi(x, y, T))+T^{-1} \frac{\partial \varphi}{\partial T}(x, y, T)=0
$$

or equivalently, the system

$$
\frac{\partial \varphi}{\partial T}(x, y, T)-\frac{a+y+\varphi(x, y, T)}{T}=0
$$

Since we know that

$$
\frac{\partial \varphi}{\partial T}(x, y, T)-\frac{a+y+\varphi(x, y, T)}{T}>0 \Longleftrightarrow T>T^{*}(x, y),
$$

this system can be solved by a simple bisection method. Hence, the overall procedure to solve the optimization problem (2.6) with a piecewise linear cost rate function has the following structure. 


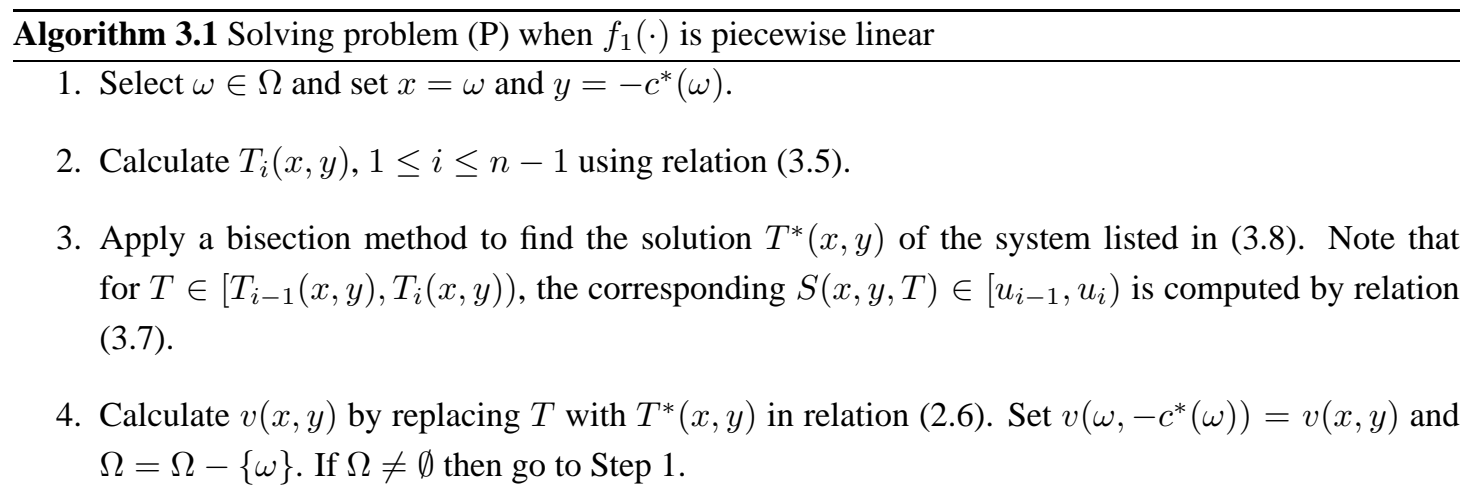

5. Calculate the optimal objective function value $v_{P}$ by relation (2.8).

As we have discussed before polyhedral concave production cost functions are frequently used in practice. Therefore, if we additionally assume that $c(\cdot)$ is polyhedral concave, the algorithm above can be further simplified as follows.

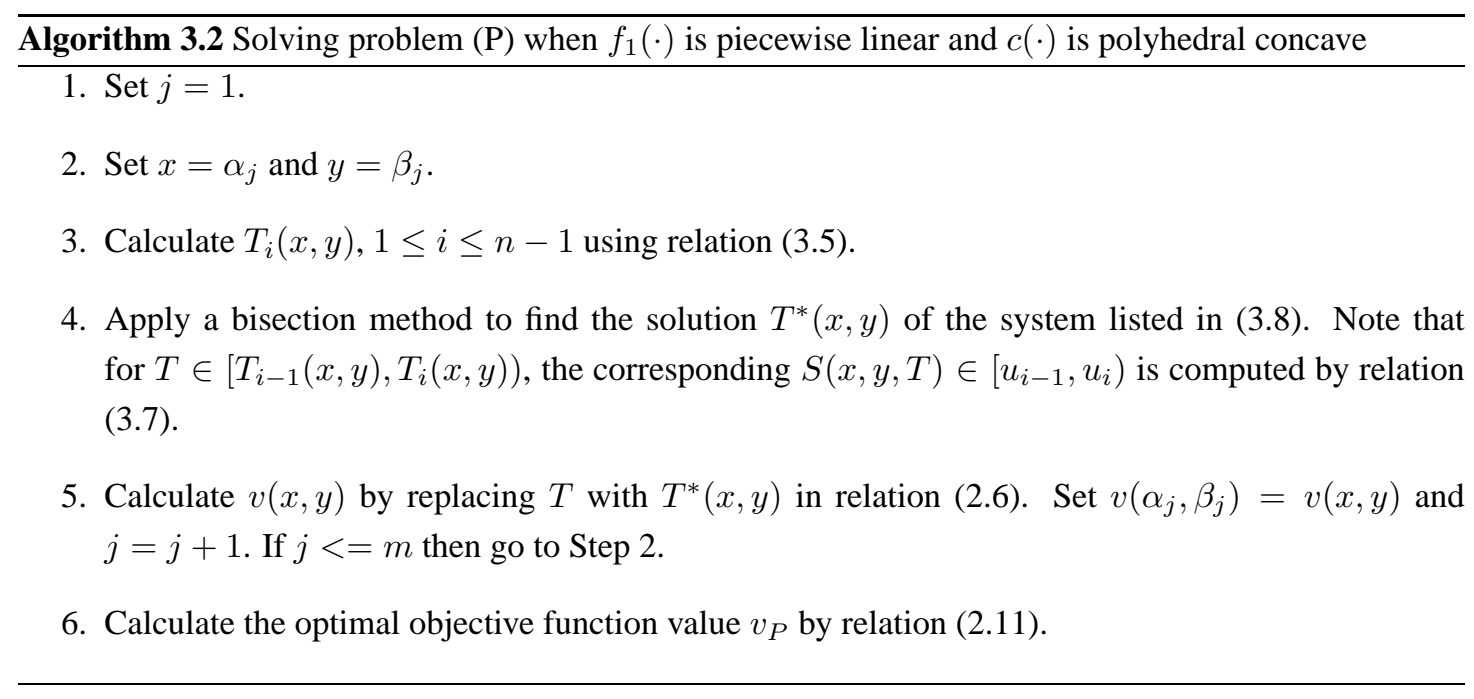

\section{Computational Results}

In this section, we illustrate that the proposed solution procedure can be easily implemented on a computer and we show that, with such an implementation, the optimal solutions can be obtained in little time. To serve our purposes, we have written a computer program for Algorithm 3.2 using MATLAB 6.1 on a Pentium III - $1 \mathrm{GHz}$ personal computer.

We have decided to focus on Algorithm 3.2 rather than Algorithm 3.1 because the majority of applications involve polyhedral concave cost functions; for instance, with incremental unit discounting, the cost functions are actually polyhedral concave, or when general concave cost functions are present, the polyhedral concave functions are used for approximation. Notice that the latter usage of the polyhedral concave functions also implies that Algorithm 3.2 can be used to approximate Algorithm 3.1.

In our experimental setting we have considered three levels for the two factors; the number of breakpoints $n$, in the piecewise linear cost rate function $f_{1}(\cdot)$ and the number of breakpoints $m$, in the poly- 
hedral cost function $c(\cdot)$. For every combination of $n$ and $m$, twenty-five problems have been randomly generated. The range of values for the other parameters as well as the levels of the two factors are given in Table 1. Note that apart from these two factors, all other randomly generated parameters are continuous. The inventory carrying charge, $r$ has been fixed to 0.2 in all problems. We have used a simple bisection method that returns a solution which is $\epsilon$ away from the actual root of the system (3.8). Each generated problem instance has been solved under three different values of the precision parameter $\epsilon ; 0.01,0.001$, 0.0001 .

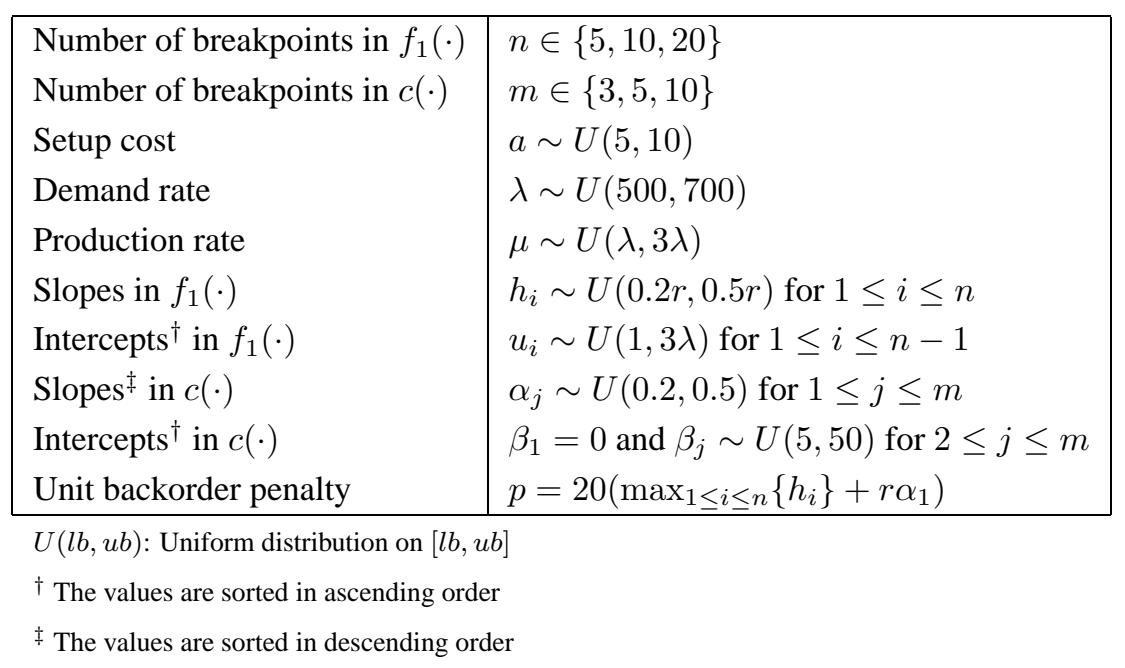

Table 1: Experimental setting for the factors and the randomly generated parameters.

Table 2 gives average running times in seconds over twenty-five runs. The figures in the table show that the solution times slightly increase as one of the two factors, $n$ and $m$ increases. When both factors are increased, then large running times are observed in the table. This is due to the computational effort invested in the double loops ( $n$ by $m$ ). Nevertheless, even for a fairly large problem, such as the one with $n=20$ and $m=10$, the procedure finds a solution almost in one-tenth of a second. On the other hand, decreasing the precision parameter $\epsilon$ has a very little effect on the running times. This is a direct consequence of using the bisection method, which converges to the root of the system exponentially fast. Overall, our observations show that in order to have better approximations, one can easily use many breakpoints and small precision parameters without a significant increase in the running time.

\begin{tabular}{|l|l|r|r|r|}
\cline { 3 - 5 } \multicolumn{1}{c}{} & $\mathbf{m}=\mathbf{3}$ & $\mathbf{m}=\mathbf{5}$ & $\mathbf{m}=\mathbf{1 0}$ \\
\hline \multirow{4}{*}{$\epsilon=\mathbf{0 . 0 1}$} & $\mathbf{n}=\mathbf{5}$ & 0.02 & 0.03 & 0.06 \\
& $\mathbf{n}=\mathbf{1 0}$ & 0.03 & 0.04 & 0.08 \\
& $\mathbf{n}=\mathbf{2 0}$ & 0.04 & 0.07 & 0.13 \\
\hline \multirow{4}{*}{$\epsilon=\mathbf{0 0 1}$} & $\mathbf{n}=\mathbf{5}$ & 0.02 & 0.03 & 0.07 \\
& $\mathbf{n}=\mathbf{1 0}$ & 0.03 & 0.04 & 0.09 \\
& $\mathbf{n}=\mathbf{2 0}$ & 0.05 & 0.08 & 0.16 \\
\hline & $\mathbf{n}=\mathbf{5}$ & 0.03 & 0.04 & 0.08 \\
& $\mathbf{n}=\mathbf{1 0}$ & 0.03 & 0.05 & 0.11 \\
& $\mathbf{n}=\mathbf{2 0}$ & 0.05 & 0.09 & 0.18 \\
\hline
\end{tabular}

Table 2: The average solution times in seconds. 


\section{References}

[1] Harris, F. How many parts to make at once. Factory, The Magazine of Management, 10:135-136, $152,1913$.

[2] Hadley, G. and T.M. Whitin. Analysis of Inventory Systems. Prentice-Hall, Englewood Cliffs, NJ, 1963.

[3] Hax, A.C. and D. Candea. Production and Inventory Management. Prentice-Hall, Englewood Cliffs, NJ, 1984.

[4] Porteus, E.L. Stochastic inventory theory. In Heyman, D.P. and M.J. Sobel, editor, Handbooks in OR and MS, volume 2. Elsevier Science Publishers, B.V., North Holland, 1990.

[5] Bayındır, Z.P., Birbil, Ş.İ and J.B.G Frenk. A multi-item inventory model with joint setup and concave production costs. Technical Report EI-2004-25, Econometric Institute, Erasmus University Rotterdam, Rotterdam, The Netherlands, 2004. http://eps.eur.nl/fewdocs/econometric_institute/109326502/.

[6] Sniedovich, M. C-programming - An outline. Operations Research Letters, 4(1):19-21, 1985.

[7] Frenk, J.B.G., Kleijn, M.J. and R. Dekker. An efficient algorithm for a generalized joint replenishment problem. European Journal of Operational Research, 118(2):413-428, 1999.

[8] Avriel, M. and Diewert, W.E. and Schaible, S. and I. Zang. Generalized Concavity. Plenum Press, New York, 1988.

[9] Hiriart-Urruty, J.B. and C. Lemaréchal. Convex Analysis and Minimization Algorithms, volume 1. Springer Verlag, Berlin, 1993.

[10] Stummel, F. and K. Hainer. Introduction to Numerical Analysis. Scottish Academic Press, 1980. 


\section{Publications in the Report Series Research* in Management}

\section{ERIM Research Program: "Business Processes, Logistics and Information Systems"}

\section{4}

Smart Pricing: Linking Pricing Decisions with Operational Insights

Moritz Fleischmann, Joseph M. Hall and David F. Pyke

ERS-2004-001-LIS

http://hdl.handle.net/1765/1114

Mobile operators as banks or vice-versa? and: the challenges of Mobile channels for banks

L-F Pau

ERS-2004-015-LIS

http://hdl.handle.net/1765/1163

Simulation-based solution of stochastic mathematical programs with complementarity constraints: Sample-path analysis S. Ilker Birbil, Gül Gürkan and Ovidiu Listeş ERS-2004-016-LIS

http://hdl.handle.net/1765/1164

Combining economic and social goals in the design of production systems by using ergonomics standards Jan Dul, Henk de Vries, Sandra Verschoof, Wietske Eveleens and Albert Feilzer

ERS-2004-020-LIS

http://hdl.handle.net/1765/1200

Factory Gate Pricing: An Analysis of the Dutch Retail Distribution

H.M. le Blanc, F. Cruijssen, H.A. Fleuren, M.B.M. de Koster

ERS-2004-023-LIS

http://hdl.handle.net/1765/1443

A Review Of Design And Control Of Automated Guided Vehicle Systems

Tuan Le-Anh and M.B.M. De Koster

ERS-2004-030-LIS

http://hdl.handle.net/1765/1323

Online Dispatching Rules For Vehicle-Based Internal Transport Systems

Tuan Le-Anh and M.B.M. De Koster

ERS-2004-031-LIS

http://hdl.handle.net/1765/1324

Generalized Fractional Programming With User Interaction

S.I. Birbil, J.B.G. Frenk and S. Zhang

ERS-2004-033-LIS

http://hdl.handle.net/1765/1325

\footnotetext{
* A complete overview of the ERIM Report Series Research in Management: https://ep.eur.nl/handle/1765/1

ERIM Research Programs:

LIS Business Processes, Logistics and Information Systems

ORG Organizing for Performance

MKT Marketing

F\&A Finance and Accounting

STR Strategy and Entrepreneurship
} 
Meta-heuristics for dynamic lot sizing: A review and comparison of solution approaches

Raf Jans and Zeger Degraeve

ERS-2004-042-LIS

http://hdl.handle.net/1765/1336

A Multi-ltem Inventory Model With Joint Setup And Concave Production Costs

Z.P. Bayındır, S.I. Birbil and J.B.G. Frenk

ERS-2004-044-LIS

http://hdl.handle.net/1765/1535

The Level Set Method Of Joó And Its Use In Minimax Theory

J.B.G. Frenk and G. Kassay

ERS-2004-045-LIS

http://hdl.handle.net/1765/1537

Reinventing Crew Scheduling At Netherlands Railways

Erwin Abbink, Matteo Fischetti, Leo Kroon, Gerrit Timmer And Michiel Vromans

ERS-2004-046-LIS

http://hdl.handle.net/1765/1427

Intense Collaboration In Globally Distributed Teams: Evolving Patterns Of Dependencies And Coordination

Kuldeep Kumar, Paul C. van Fenema and Mary Ann Von Glinow

ERS-2004-052-LIS

http://hdl.handle.net/1765/1446

The Value Of Information In Reverse Logistics

Michael E. Ketzenberg, Erwin van der Laan and Ruud H. Teunter

ERS-2004-053-LIS

http://hdl.handle.net/1765/1447

Cargo Revenue Management: Bid-Prices For A 0-1 Multi Knapsack Problem

Kevin Pak and Rommert Dekker

ERS-2004-055-LIS

http://hdl.handle.net/1765/1449

Real-Time Scheduling Approaches For Vehicle-Based Internal Transport Systems

Tuan Le-Anh and M.B.M. De Koster

ERS-2004-056-LIS

http://hdl.handle.net/1765/1452

Activating Knowledge Through Electronic Collaboration: Vanquishing The Knowledge Paradox

S. Qureshi and P. Keen

ERS-2004-058-LIS

http://hdl.handle.net/1765/1473

A Grounded Theory Analysis Of E-Collaboration Effects For Distributed Project Management

S. Qureshi, M. Liu and D. Vogel

ERS-2004-059-LIS

http://hdl.handle.net/1765/1448

Collaborative Infrastructures For Mobilizing Intellectual Resources: Assessing Intellectual Bandwidth In A

Knowledge Intensive Organization

R. Verhoef and S. Qureshi

ERS-2004-060-LIS

http://hdl.handle.net/1765/1474

Satisfaction Attainment Theory As A Model For Value Creation

R.O. Briggs, S. Qureshi and B. Reining

ERS-2004-062-LIS

http://hdl.handle.net/1765/1450 
Diagnosis In The Olap Context

Emiel Caron, Hennie Daniels

ERS-2004-063-LIS

http://hdl.handle.net/1765/1492

A Deterministic Inventory/Production Model With General Inventory Cost Rate Function And Concave Production Costs Z.P. Bayındır, S.I. Birbil and J.B.G. Frenk

ERS-2004-064-LIS

http://hdl.handle.net/1765/1536 